\title{
SINAIS E SINTOMAS RELACIONADOS À EXPOSIÇÃO À FUMAÇA CIRÚRGICA: REVISÃO INTEGRATIVA
}

\author{
Signs and symptoms related to surgical smoke exposure: integrative review
}

\section{Signos y síntomas relacionados con la exposición al humo quirúrgico: revisión integrativa}

\author{
Aryane Apolinario Bieniek* (D), Patricia Aroni² (D), Raquel Gvozd Costa ${ }^{3}$ (D), Renata Perfeito Ribeiro ${ }^{4}$ (I)
}

RESUMO: Objetivo: Analisar as evidências científicas disponíveis na literatura sobre os sinais e sintomas relacionados à exposição à fumaça cirúrgica em trabalhadores do bloco operatório. Método: Revisão integrativa da literatura, com busca nas seguintes bases de dados: Medline, Cumulative Index to Nursing and Allied Health Literature, Literatura Latino-Americana e do Caribe em Ciências da Saúde, Web Of Science e SCOPUS. Resultados: Foram 1.351 estudos pré-selecionados, sendo 4 por busca manual. Ao fim, obtiveram-se cinco artigos para análise. Os sinais e sintomas encontrados foram: tosse, ardência de faringe, espirros, rinite, lesão nasofaringe, sensação de corpo estranho na garganta, congestão nasal, inflamação das vias aéreas, lacrimejamento dos olhos, náuseas, vômitos, dor abdominal, fraqueza, cãibra, cefaleia, sonolência, tonturas, irritabilidade, desconforto (como mau cheiro na roupa). Conclusão: Observaram-se grandes avanços nas pesquisas propostas, estudos que embasam a intensidade dos sinais e sintomas e orientação dos riscos nocivos que proporcionem aos gestores conhecimento e fundamentação científica para futuras intervenções tanto contra a proteção ao trabalhador como para prevenção de risco no ambiente de trabalho. Palavras-chave: Sinais e sintomas. Fumaça. Eletrocirurgia. Eletrocoagulação. Saúde do trabalhador.

ABSTRACT: Objective: To analyze the scientific evidence available in the literature on signs and symptoms related to surgical smoke exposure among surgical block workers. Method: This integrative literature review searched the following databases: MEDLINE, Cumulative Index to Nursing and Allied Health Literature, Latin American and Caribbean Health Sciences Literature, Web of Science, and Scopus. Results: A total of 1,351 studies were pre-selected, 4 of which were found by manual search. In the end, five articles were analyzed. The signs and symptoms identified were: cough, burning sensation in the pharynx, sneezing, rhinitis, nasopharyngeal lesion, foreign body sensation in the throat, nasal congestion, airway inflammation, lacrimation, nausea, vomiting, abdominal pain, weakness, muscle cramp, headache, drowsiness, lightheadedness, irritability, discomfort (such as unpleasant smell in clothes). Conclusion: Great advances have been detected in the studies proposed. These investigations lay the foundation for the intensity of signs and symptoms and the orientation regarding harmful risks, providing managers with the knowledge and scientific basis for future interventions, both to protect the team and to prevent risks in the work environment. Keywords: Signs and symptoms. Smoke. Electrosurgery. Electrocoagulation. Occupational health.

RESUMEN: Objetivo: Analizar la evidencia científica disponible en la literatura sobre signos y síntomas relacionados con la exposición al humo quirúrgico en trabajadores de quirófano. Método: Revisión integrativa de la literatura, buscando en las siguientes bases de datos: Medline, Cumulative Index to Nursing and Allied Health Literature, Latin American and Caribbean Literature in Health Sciences, Web Of Science y SCOPUS. Resultados: Hubo 1.351 estudios preseleccionados, cuatro por búsqueda manual. Al final, se obtuvieron cinco artículos para su análisis. Los signos y síntomas encontrados fueron: tos, ardor faríngeo, estornudos, rinitis, lesión nasofaríngea, sensación de cuerpo extraño en la garganta, congestión nasal, inflamación de las vías respiratorias, lagrimeo de los ojos, náuseas, vómitos, dolor abdominal, debilidad, calambres, dolor de cabeza, somnolencia, mareos, irritabilidad, malestar como mal olor en la ropa. Conclusión: Hubo grandes avances en la investigación propuesta, estudios que apoyan la intensidad de los signos y síntomas y orientaciones sobre riesgos nocivos que brinden a los gestores conocimiento y fundamento científico para futuras intervenciones, tanto contra la protección del trabajador como para la prevención de riesgos en el entorno laboral. Palabras clave: Signos y síntomas. Humo. Electrocirugia. Eletrocoagulación. Salud laboral.

'Doutoranda do Programa de Pós-Graduação em Enfermagem Fundamental da Universidade Estadual de Londrina (UEL). Enfermeira em Centro de Material Esterilizado no Hospital Reginal do Norte do Paraná (HU) - Londrina (PR), Brasil.

${ }^{2}$ Doutora em Enfermagem pela Escola de Enfermagem de Ribeirão Preto da Universidade de São Paulo (EERP-USP). Docente do Departamento de Enfermagem da UEL na área de gerência de enfermagem 'Doutora em Enfer

${ }^{3}$ Doutora em Enfermagem pela Universidade Estadual de Maringá. Docente do departamento de Enfermagem da UEL na área de gerência de enfermagem - Londrina (PR), Brasil.

«Pós-Doutorada em Enfermagem pela EERP-USP. Docente do Departamento de Enfermagem da UEL na área de gerência de Enfermagem. Coordenadora do Núcleo de Pesquisa em Saúde do Trabalhador da Universidade Estadual de Londrina - Londrina (PR), Brasil.

*Autora correspondente: aryanebieniek@gmail.com

Recebido: 30/11/2020 - Aprovado: 19/08/2021

https://doi.org/10.5327/Z1414-4425202100030009 


\section{INTRODUÇÃO}

O eletrocautério é um aparelho eletrônico de alta frequência, capaz de promover efeitos como corte, dissecção e coagulação em tecidos e vasos. Seu uso é frequente em diferentes procedimentos cirúrgicos, dadas as suas vantagens, que, além de reduzir o risco de hemorragias, também facilita a visualização do campo cirúrgico e diminui o tempo das cirurgias ${ }^{1}$.

No momento em que o eletrocautério entra em combustão com o tecido, forma-se uma fumaça cirúrgica, na qual foi identificada a presença de compostos químicos tóxicos, com potencial cancerígeno, como formaldeído, cianeto de hidrogênio, benzeno e monóxido de carbono, além de material celular e partículas de vírus².

A presença desses compostos na fumaça cirúrgica traz riscos aos trabalhadores expostos durante os procedimentos cirúrgicos, com complicações para a sua saúde, mesmo quando estes fazem uso de equipamentos de proteção individual (EPI), tais como a máscara cirúrgica e os óculos ${ }^{3}$, como acontece, de forma geral, nos centros cirúrgicos (CC).

Uma revisão da literatura acerca da utilização do bisturi elétrico e dos cuidados de enfermagem relacionados ao seu uso identificou o desconhecimento por parte dos funcionários e enfermeiros quanto ao seu manuseio e funcionamento ${ }^{4}, \mathrm{o}$ qual interfere no risco à saúde do trabalhador.

Em estudo realizado num hospital no México, com 115 médicos residentes de todas as especialidades cirúrgicas, onde o eletrocautério é utilizado em $70 \%$ dos procedimentos cirúrgicos, os autores encontraram os seguintes sinais e sintomas: sensação de corpo estranho na garganta, ardência na faringe, náuseas, vômitos, congestão nasal, cefaleia, irritação dos olhos e outras mucosas, espirros, fraqueza e tontura ${ }^{1}$.

Neste estudo, entendeu-se a necessidade de definir sinal e sintoma, em que sinal é um dado objetivo relatado, ou seja, uma queixa referida e identificada; já sintoma indica uma sensação subjetiva de anormalidade ${ }^{5}$.

De acordo com a experiência dos pesquisadores deste estudo, percebe-se, na prática, que entre os trabalhadores existe o relato de outros sinais e sintomas, além desses encontrados. Identifica-se que a explanação desses sinais e sintomas auxilia na compreensão dos riscos envolvidos com a exposição dos profissionais de saúde à fumaça cirúrgica. Além disso, esses resultados têm potencial para auxiliar na elaboração de estratégias de melhoria para a saúde desse trabalhador. Portanto, há necessidade de uma busca sistemática por mais achados científicos que envolvam essa temática.

\section{OBJETIVO}

Analisar as evidências científicas disponíveis na literatura sobre os sinais e sintomas relacionados à exposição à fumaça cirúrgica em trabalhadores do bloco operatório.

\section{MÉTODO}

\section{Desenho do estudo}

O presente estudo é uma revisão integrativa da literatura que reúne e sintetiza o conhecimento produzido por diversos estudos, além de apontar lacunas do conhecimento da temática para serem descobertas em novas pesquisas ${ }^{6}$.

As seguintes fases nortearam o estudo: escolha do tema e determinação do objetivo, elaboração da pergunta norteadora da pesquisa, busca em bases de dados para identificar pesquisas primárias nos critérios de elegibilidade dos estudos e, ao fim do processo, realização da análise crítica dos estudos incluídos ${ }^{6}$.

\section{Identificação do tema e seleção da hipótese ou questão de pesquisa para elaborar a revisão integrativa}

Para guiar a revisão, apresentou-se a seguinte pergunta de pesquisa ao presente estudo: quais são as consequências para os trabalhadores da saúde expostos à fumaça cirúrgica, em relação aos sinais e sintomas apresentados?

Para elaborar a pergunta de pesquisa, utilizou-se a estratégia PICO, uma sigla que consiste em: "P" de pacients, os quais, neste estudo, foram "trabalhadores da saúde"; "I" de interventions, que foi "eletrocautério"; "O" do inglês outcomes, que significa desfecho clínico ou resultado, o qual foi representado por "consequências referentes aos sinais e sintomas"; "C" de comparativo, que não se aplica ao objetivo do estudo?.

Neste estudo, utilizaram-se os termos sinal e sintoma, sendo considerado sinal quando um dado relatado for objetivo, ou seja, reflete uma queixa referida e identificada; sintoma é considerado quando o dado relatado for uma sensação subjetiva de anormalidade 5 .

\section{Procedimento de coleta de dados}

As bases de dados selecionadas para a busca dos estudos primários foram: Scopus, National Library of Medicine National 
Institutes of Health (Medline) via PubMed, Cumulative Index to Nursing and Allied Health Literature (CINAHL), Literatura Latino-Americana e do Caribe em Ciências da Saúde (LILACS) e Web Of Science (WOS). Além disso, incluíram-se na análise desta revisão estudos adquiridos por busca manual que fazem parte do banco de dados do Núcleo de Estudos em Saúde do Trabalhador da Universidade Estadual de Londrina (NUESTUEL).

$\mathrm{Na}$ estratégia para busca dos estudos nas bases de dados, utilizaram-se os descritores controlados presentes nos Descritores em Ciências da Saúde (DeCS): eletrocirurgia, eletrocoagulação, saúde do trabalhador; e no Medical Subject Headings (MeSH): electrosurgery, electrocoagulation, surgical smoke, symptoms and signs e health occupation. Os descritores não controlados nos DeCS foram: fumaça cirúrgica, fumaça, terapia a laser, cauterização, sinais e sintomas, exposição ocupacional, segurança do trabalho, segurança ocupacional, bisturi a laser, eletrocautério, manifestações clínicas, sinais clínicos, queixas e sintomas, exposição laboral a agentes químicos, físicos ou biológicos; e no MeSh: smoke, laser therapy, cautery, electrocautery, smoke evacuation, thermocoagulation, galvanocautery, surgical diathermy, endocavitary fulguration, personnel health, healthcare worker e health professions.

Para o cruzamento entre os termos, utilizaram-se os operadores boleanos "AND" e "OR", com a finalidade de obter uma busca refinada e maior número de artigos possíveis para responder à pergunta norteadora. A busca foi realizada no período de maio a junho de 2018 .

Fez-se uma busca ampla da literatura descrita, não havendo limitação de idioma ou de ano de publicação. Incluíram-se nesta pesquisa somente estudos primários.

\section{Critérios de seleção}

A seleção dos artigos foi realizada primeiramente pela leitura dos títulos e resumos e, posteriormente, dos textos na íntegra. O processo de seleção dos artigos foi feito por dois revisores independentes, e, em caso de dúvidas, um terceiro revisor reavaliou.

\section{Análise e tratamento dos dados}

Para avaliar o nível de evidência dos artigos, considerou-se:

- nível 1: evidência de uma revisão sistemática de todos os ensaios clínicos randomizados relevantes ou diretrizes de prática clínica baseadas em evidências de revisões sistemáticas;
- nível 2: evidência obtida de pelo menos um ensaio clínico randomizado, bem delineado;

- nível 3: evidências obtida por ensaios clínicos controlados bem desenhados, sem randomização e estudos quase-experimentais;

- nível 4: evidências de estudos de caso controle e coorte bem desenhado;

- nível 5: evidências de revisões sistemáticas de estudos descritivos e qualitativos;

- nível 6: evidências de um único descritivo ou estudos qualitativos;

- nível 7: evidências de opiniões de autoridades e/ou relatórios de comitê de especialista ${ }^{7}$.

Nesta revisão, não se incluíram artigos com nível de evidência 1,5 e 7.

Este estudo seguiu as etapas propostas pelo checklist Preferred Reporting Items for Systematic Reviews and MetaAnalyses (PRISMA).

A Figura 1 representa o fluxograma no qual se demonstra o caminho percorrido para classificação, seleção e inclusão dos estudos primários, segundo as bases de dados elencadas.

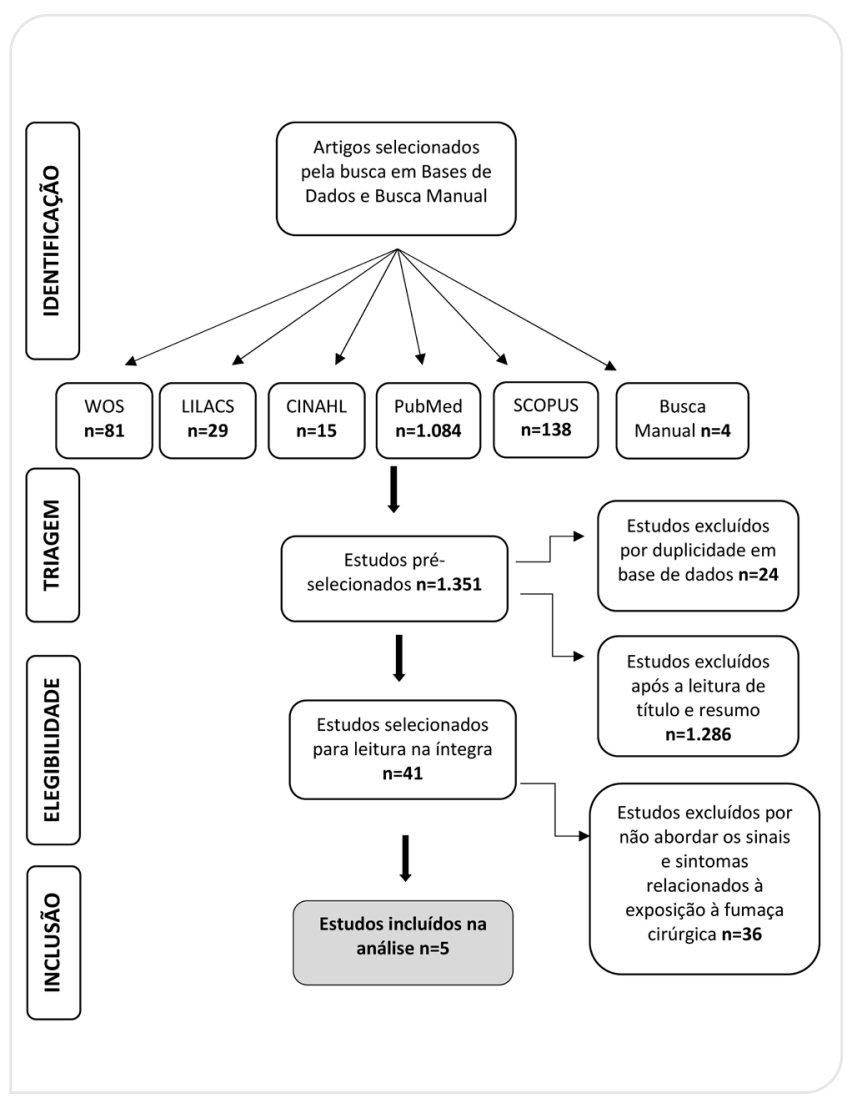

Figura 1. Fluxograma dos estudos da revisão integrativa. 


\section{RESULTADOS}

Para apresentar os resultados, optou-se por identificar os estudos pela letra E, de estudo, com sequência numérica de um a cinco. Dos cinco estudos primários selecionados, todos foram publicados nos últimos 12 anos, três estudos estavam no idioma turco (60\%), dois em inglês (40\%) e um em espanhol (20\%).

Quanto ao delineamento dos estudos, sua totalidade foi classificada como estudos observacionais, com característica descritiva e abordagem quantitativa, sendo assim, com evidência científica Nível 6.

Quadro 1. Síntese dos estudos selecionados, segundo autores, país, população e sinais e sintomas relacionados à exposição à fumaça cirúrgica.

\begin{tabular}{|c|c|c|}
\hline Estudo & População / Amostra & Sinais e Sintomas \\
\hline $\begin{array}{l}\text { E1 - Navarro-Meza et al., 2013' } \\
\text { (México) }\end{array}$ & $\begin{array}{l}\text { Médicos residentes: } \\
50\end{array}$ & $\begin{array}{l}\text { - Sensação de corpo estranho na garganta: } 58,0 \% \\
\text { - Ardência na faringe: } 22,0 \% \\
\text { - Náusea: } 4,0 \% \\
\text { - Congestão nasal: } 2,0 \%\end{array}$ \\
\hline $\begin{array}{l}\text { E2 - llce et al., } 2017^{9} \\
\text { (Turquia) }\end{array}$ & $\begin{array}{l}\text { Enfermeiros: } 45 \\
\text { Médicos: } 36\end{array}$ & $\begin{array}{l}\text { - Cefaleia (enfermeiros: } 48,9 \% \text {, médicos: } 58,3 \% \text { ) } \\
\text { - Lacrimejamento (enfermeiros: } 40,0 \% \text {, médicos: } 41,7 \% \text { ) } \\
\text { - Tosse (enfermeiros: } 48,9 \% \text {, médicos: } 27,8 \% \text { ) } \\
\text { - Ardência de faringe (enfermeiros } 40,0 \% \text {, médicos: } 38,9 \% \text { ) } \\
\text { - Náusea (enfermeiros } 44,4 \% \text {, médicos: } 30,6 \% \text { ) } \\
\text { - Outros* }\end{array}$ \\
\hline $\begin{array}{l}\text { E3 - Ünver et al., } 2016^{10} \\
\text { (Turquia) }\end{array}$ & $\begin{array}{l}\text { Enfermeiros: } \\
\qquad 54\end{array}$ & $\begin{array}{l}\text { - Cefaleia: } 59,3 \% \\
\text { - Irritação na faringe: } 56,6 \% \\
\text { - Náusea: } 40,7 \% \\
\text { - Olhos lagrimejantes: } 38,9 \% \\
\text { - Fraqueza: } 24,1 \% \\
\text { - Tontura: } 9,3 \%\end{array}$ \\
\hline $\begin{array}{l}\text { E4 - Usta et al., } 2019^{11} \\
\text { (Turquia) }\end{array}$ & $\begin{array}{l}\text { Enfermeiros: } \\
\qquad 105\end{array}$ & $\begin{array}{l}\text { - Cefaleia: } 61,9 \% \\
\text { - Náusea: } 39 \% \\
\text { - Vômito: } 14,3 \% \\
\text { - Tosse: } 41,0 \% \\
\text { - Irritação no olho: } 54,3 \% \\
\text { - Ardência na faringe: } 43,8 \% \\
\text { - Irritabilidade: } 29,5 \% \\
\text { - Tonturas: } 32,4 \% \\
\text { - Problemas respiratórios: 27,6\% } \\
\text { - Lesão de nasofaringe: 7,6\% } \\
\text { - Fraqueza: } 25,7 \% \\
\text { - Cãibra: } 22,9 \% \\
\text { - Dor abdominal: } 15,2 \%\end{array}$ \\
\hline $\begin{array}{l}\text { E5 - Okgün Alcan et al., } 2017^{12} \\
\text { (Turquia) }\end{array}$ & $\begin{array}{l}\text { Enfermeiros: } \\
\qquad 71\end{array}$ & $\begin{array}{l}\text { - Cefaleia: } 71,8 \% \\
\text { - Náusea: } 63,4 \% \\
\text { - Tosse: } 57,7 \% \\
\text { - Ardência na faríngea: } 49,3 \% \\
\text { - Lacrimejamento: } 46,5 \% \\
\text { - Espirro: } 39,4 \% \\
\text { - Tontura: } 38,0 \% \\
\text { - Irritabilidade: } 31,0 \% \\
\text { - Inflamação das vias aéreas: } 29,6 \% \\
\text { - Fraqueza: } 28,2 \% \\
\text { - Lesão de nasofaringe: } 7,0 \% \\
\text { - Vômitos: } 5,6 \% \\
\text { - Dor abdominal: } 8,5 \% \\
\text { - Cãibra: } 9,9 \%\end{array}$ \\
\hline
\end{tabular}

*Outros: sonolência, tonturas, espirros, fraqueza, irritação, lesão de nasofaringe, dor abdominal, vômitos. 
No Quadro 1, apresenta-se a síntese dos estudos selecionados para compor esta revisão, segundo autores, país, população e sinais e sintomas relacionados à exposição à fumaça cirúrgica.

Além dos sinais e sintomas apresentados no Quadro 1, alguns estudos trouxeram doenças relacionadas à exposição à fumaça cirúrgica, como: anemia, rinite, asma, conjuntivite, dermatite, doenças cardiovasculares, hepatite e câncer.

Na Tabela 1, apresentam-se os sinais e sintomas relacionados à exposição à fumaça cirúrgica.

Nos cinco estudos da revisão, na maioria dos artigos, o sistema respiratório foi o que apresentou maior incidência de sinais e sintomas, quando o profissional é exposto à fumaça cirúrgica, seguido do sistema ocular.

\section{DISCUSSÃO}

Um estudo descritivo, no qual os autores analisaram os subprodutos gasosos presentes na fumaça cirúrgica, verificou que, por seu tamanho aerodinâmico, as partículas geradas, inaladas pelos trabalhadores da área da saúde e pacientes, percorrem uma distância de até $100 \mathrm{~cm}$ do local produzido ${ }^{13}$. Verificou-se, também, que $1 \mathrm{~g}$ de tecido cauterizado provoca uma nuvem de fumaça cirúrgica com efeitos mutagênicos, sendo equivalente a fumar de três a seis cigarros sem filtro ${ }^{14,15}$.

Além disso, a maior incidência de sinais e sintomas, encontrados neste estudo, está associada ao sistema respiratório. Esse resultado pode estar ligado ao fato de que a inalação da fumaça cirúrgica pode penetrar no pulmão, acarretando doenças crônicas e agudas, como: congestionamento alveolar, pneumonia intersticial e bronquiolite ${ }^{13}$.

O mesmo estudo informa que, dos produtos químicos preocupantes mais encontrados na fumaça cirúrgica, o acrilonitrilo e o monóxido de carbono (CO) são responsáveis pelos sinais e sintomas de irritação nos olhos, náuseas, vômitos, cefaleia, espirros, fraqueza e tontura, e a exposição prolongada a eles pode produzir irritação e dermatite. Esses sinais e sintomas estão relacionados aos sistemas respiratório e tegumentar ${ }^{13}$.

Em conceito fisiológico, o processo de trocas gasosas ocorre na ventilação e na inalação do ar para o interior dos alvéolos, os quais são revestidos por vasos capilares, onde a troca ocorre na interface entre o epitélio alveolar, o interstício e o capilar tecidual ${ }^{16}$. Sendo assim, o sistema respiratório, quando exposto à fumaça cirúrgica, torna-se o alvo principal, provavelmente pelo tamanho das partículas oxidantes presentes na fumaça cirúrgica.

As partículas dissipadas no ambiente com diâmetro menor que $10 \mu \mathrm{m}$ são inaláveis e com potencial de comprometimento do trato respiratório; já as partículas inferiores a 2,5 $\mu \mathrm{m}$, quando inaladas, atingem os alvéolos pulmonares; partículas com diâmetros ainda menores a $0,01 \mu \mathrm{m}$, caracterizadas como partículas ultrafinas (UFP), dispostas na fumaça cirúrgica, possuem penetração mais profunda no sistema respiratório, levando ao maior comprometimento desse sistema ${ }^{1,17-20}$

O composto químico acrinolitrilo, presente na nevoa cirúrgica, tem seu efeito tóxico quando inalado, pela formação de cianeto, responsável pelos incômodos como irritação nos olhos, náuseas, vômitos, cefaleia, espirros, fraqueza e tontura. Quando em uso prolongado, esse composto tem potencial cancerígeno e causa irritação e dermatite ${ }^{13}$.

Já o produto químico $\mathrm{CO}$, mais encontrado em procedimentos laparoscópicos, é absorvido pelas células e encaminhado à corrente sanguínea. Em combinação com a hemoglobina, desencadeia uma resposta sistêmica de estresse hipóxico, resultando na diminuição da capacidade de transporte de oxigênio ao corpo. Em indivíduos com doenças cardiovasculares, pode ser ainda mais prejudicial à saúde ${ }^{13,21}$.

A característica do produto químico está diretamente ligada ao tipo de tecido onde foi realizada a intervenção com o eletrocautério. Nos tecidos adiposos, há maior produção

Tabela 1. Sinais e sintomas divididos por sistemas, identificados nos cinco estudos selecionados para esta revisão.

\begin{tabular}{|c|c|c|c|c|c|c|}
\hline Estudos da & $\begin{array}{l}\text { Sinais e } \\
\text { sintomas }\end{array}$ & $\begin{array}{c}\text { Sistema } \\
\text { respira-tório }\end{array}$ & Sistema ocular & $\begin{array}{c}\text { Sistema } \\
\text { digestório }\end{array}$ & $\begin{array}{c}\text { Sistema } \\
\text { osteomuscular }\end{array}$ & $\begin{array}{l}\text { Sistema } \\
\text { nervoso }\end{array}$ \\
\hline & $\mathbf{n}$ & n (\%) & n (\%) & n (\%) & n (\%) & n (\%) \\
\hline E1 & 04 & $03(75,0)$ & - & $01(25,0)$ & - & - \\
\hline E2 & 13 & $04(30,8)$ & $01(7,7)$ & $03(23,0)$ & $01(7,7)$ & $04(30,8)$ \\
\hline E3 & 06 & $01(16,6)$ & $01(16,6)$ & $01(16,6)$ & $01(16,6)$ & $02(33,2)$ \\
\hline E4 & 13 & $04(30,8)$ & $01(7,8)$ & $03(23,0)$ & $02(15,4)$ & $03(23,0)$ \\
\hline E5 & 14 & $04(28,6)$ & $02(14,3)$ & $03(21,4)$ & $02(14,3)$ & $03(21,4)$ \\
\hline
\end{tabular}


de aldeídos e menos de tolueno e, consequentemente, maior desenvolvimento de sinais e sintomas ${ }^{22}$.

Em caso comprovado envolvendo um cirurgião relata-se que, após o tratamento de um paciente com condiloma anogenital com uso do laser, o cirurgião desenvolveu papilomatose laríngea. As investigações foram realizadas e identificaram a presença de papilomavírus humano (HPV) do tipo 6 e 11 no paciente, os mesmos encontrados na laringe do cirurgião; não havendo outra possibilidade de desencadear essa doença, foi representado o risco ocupacional a esse trabalhador ${ }^{23}$. Quanto ao tempo de exposição para seu desenvolvimento, não se sabe ao certo, porém a literatura nos informa que o período de incubação do HPV pode variar de dois a oito meses, podendo levar até 20 anos para o desenvolvimento de sinais e sintomas clínicos ${ }^{24}$.

Dos artigos analisados nesta revisão, nenhum deles descreveu a presença de sistema de evacuação de fumaça cirúrgica nas salas operatórias, como recomendado pelas organizações mundiais. Em alguns estudos, uso-se cateter de aspiração para aspirar a fumaça cirúrgica, o qual não se evidencia como proteção contra os riscos. Em um dos estudos, os autores relataram que $92,5 \%$ dos trabalhadores sabiam sobre a existência da fumaça cirúrgica e 55,6\% tinham conhecimento do risco nocivo quando expostos a essas substâncias ${ }^{12,25}$.

As doenças apresentadas, por consequência da inalação da fumaça cirúrgica, são: enfisema, asma, bronquite, dermatites, disfunção cardiovascular, anemia, leucemia, lesão em face nasal, infecção pelo vírus da imunodeficiência humana (HIV) e hepatites. A fumaça cirúrgica é cumulativa no organismo humano, de modo que, quanto mais tempo de exposição, maior o risco ${ }^{13,26}$.

Há diversos efeitos prejudiciais relatados na literatura em relação à fumaça cirúrgica; no entanto, existe uma lacuna de evidências e recomendações uniformes para a proteção dos trabalhadores contra exposição à fumaça cirúrgica ${ }^{27}$.

Como recomendações, a Occupational Safety and Health Administration (OSHA) orienta o uso das máscaras cirúrgicas para capturar as partículas de dimensões maiores que $5 \mathrm{~mm}$. Esse tipo de máscara não fornece proteção contra a filtração de todos os compostos presentes na fumaça cirúrgica, pela não vedação e inexistência de filtro nessa máscara. Assim, a máscara cirúrgica não está adequada para proteger contra a fumaça cirúrgica ${ }^{13,27}$.

Quanto à evacuação da fumaça cirúrgica para a OSHA, não há especificações sobre a filtração por equipamentos, porém outras organizações, por exemplo, a Association of periOperative Registered Nurses (AORN), recomendam o sistema de filtração da fumaça cirúrgica e aerossóis em altas concentrações ${ }^{25,28}$. As demais recomendações incluem o controle de engenharia, ou seja, a ventilação das salas operatórias: devem ocorrer 20 trocas de ar por cada hora ${ }^{29}$.

O sistema de evacuação de fumaça e os dispositivos devem ser utilizados em concordância com as recomendações do fabricante. Em altas concentrações de fumaça cirúrgica de partículas ultrafinas menores que $0,01 \mu \mathrm{m}$, recomenda-se o uso de filtros para o evacuador de fumaça. Sendo assim, para pequenas concentrações de fumaça, o trabalhador deve utilizar sistema de vácuo cirúrgico com um filtro $0,1 \mu \mathrm{m}$ instalado entre a conexão da parede de sucção e o recipiente de sucção. Portanto o dispositivo de captura de fumaça deve estar o mais próximo possível do local cirúrgico, para coleta da fumaça cirúrgica, nociva à saúde do trabalhador ${ }^{30}$.

Para diminuir o risco ocupacional aos trabalhadores expostos à fumaça cirúrgica, Guideline recomenda o uso de um conjunto de medidas de proteção, como sistemas de evacuação para a fumaça cirúrgica com a filtração do ar e o uso de máscara que proteja contra partículas de diâmetro $0,01 \mu \mathrm{m}$, ou seja, máscara com filtragem de $95 \%$ das partículas, conhecida como $\mathrm{N} 95^{25}$.

As recomendações do uso da N95 como uma das medidas de proteção podem proteger os trabalhadores expostos à fumaça cirúrgica. Contudo o cumprimento dessa medida é précario, pelo incômodo uso da máscara com filtragem de 95\%. Consequentemente, não há reconhecimento pelos trabalhadores quanto ao risco à exposição à fumaça cirúrgica ${ }^{31}$.

$\mathrm{E}$, ainda, precisa-se levar em consideração que a falta de reconhecimento do risco, o incômodo quanto ao uso dos EPI por parte dos trabalhadores ${ }^{31} \mathrm{e}$ a falta de estudos que indiquem as melhores formas de prevenção a esse risco dificultam a prevenção do adoecimento entre os trabalhadores da área da saúde.

Em relação às limitações deste estudo, ressalta-se a falta de padronização de instrumentos para coleta de dados dos estudos selecionados desta revisão, como também os sinais e sintomas relacionados à exposição à fumaça cirúrgica, pois, na maioria dos artigos, estes eram confundidos com doenças. Dessa forma, há necessidade de estudos com evidências científicas fortes, os quais oferecerão subsídios a futuros estudos para embasamento dessa temática. Há uma lacuna do conhecimento quanto ao tempo de exposição à fumaça cirúrgica para o desenvolvimento dos sinais e sintomas clínicos. Novas descobertas têm sido desbravadas, porém não existe nenhum consenso quanto ao tempo de exposição. 
Destaca-se, também, a escassez de estudos com rigor cientifico, a qual qualifica as pesquisas como fortes estudos para uma discussão fundamentada.

\section{CONCLUSÃO}

Ante o objetivo do presente estudo, a consequência ao trabalhador exposto à fumaça cirúrgica é o desenvolvimento dos sinais e sintomas encontrados nesta revisão, como: tosse, ardência de faringe, espirros, rinite, lesão de nasofaringe, sensação de corpo estranho na garganta, congestão nasal, inflamação das vias aéreas, lacrimejamento dos olhos, náuseas, vômitos, dor abdominal, fraqueza, cãibra, dermatite, cefaleia, sonolência, tonturas, irritabilidade, desconforto (como mau cheiro na roupa). Destacam-se, ainda, algumas doenças, como: anemia, rinite, conjuntivite, doenças cardiovasculares, hepatite e câncer.

Esperam-se grandes avanços nas pesquisas sobre a temática com o desenvolvimento deste estudo, pesquisas que embasem a intensidade dos sinais e sintomas desencadeados em trabalhadores quando expostos à fumaça cirúrgica e, consequentemente, orientação dos riscos nocivos que proporcionem aos gestores conhecimento e fundamentação científica para futuras intervenções tanto contra a proteção ao trabalhador como para prevenção de risco no ambiente onde trabalham.

\section{REFERÊNCIAS}

1. Navarro-Meza M, González-Baltazar R, Alabama-Rodríguez MG, Carmona-Navarro DE, López-Cardona MG. Síntomas respiratórios usados pelo uso do eletrocauterio em médicos na formação cirúrgica de um hospital do México. Rev Peru Med Exp. Salud Publica [Internet]. 2013 [acessado em 10 jun. 2019];30(1):41-4. Disponivel em: http://www.scielo.org.pe/scielo. php?script=sci_arttext\&pid=S1726-46342013000100008\&lng=es

2. Kalil J, Pessine FBT, Fidelis CHV, Menezes FH, Palma PCR. Analysis, cromatografia / espectrometria de massas, fumaça gerada por eletrocautério. Rev Col Bras Cir [Internet]. 2016 [acessado em 10 jun. 2019];43(2):124-8. Disponível em: https://doi. org/10.1590/0100-69912016002009

3. Bree K, Barnhill S, Rundell W. The dangers of electrosurgical smoke to operating room personnel: a review. Workplace Health Saf [Internet]. 2017 [acessado em 10 jun. 2019];65(11):517-26. Disponível em: https://doi.org/10.1177/2165079917691063

4. Olímpio M, Sousa V, Ponte M. 0 uso do bisturi elétrico e cuidados relacionados: revisão integrativa. Rev SOBECC. 2016;21(3):154-61. https://doi.org/10.5327/Z1414-4425201600030006

5. Souza Porto MF. Análise de riscos nos locais de trabalho: conhecer para transformar. São Paulo: Instituto Nacional de Saúde no Trabalho; 2000.

6. Polit DF, Beck CT. Essentials of nursing research: methods, appraisal, and utilization. $6^{\text {a }}$ ed. Filadélfia: Lippincott Williams \& Wilkins; 2005.

7. Melnyk BM, Fineout-Overholt E. Evidence-based practice in nursing $\&$ healthcare: a guide to best practice. $2^{\mathrm{a}}$ ed. Filadélfia: Lippincot Williams \& Wilkins; 2011

8. Galvão TF, Pansani TSA, Harrad D. Principais itens para relatar revisões sistemáticas e meta-análises: a recomendação PRISMA. Epidemiol Serv Saúde. 2015;24(2):335-42. https://doi.org/10.5123/ S1679-49742015000200017

9. Ilce A, Yuzden GE, Yavuz van Giersbergen M. The examination of problems experienced by nurses and doctors associated with exposure to surgical smoke and the necessary precautions. J Clin Nurs. 2017;26(11-12):1555-61. https://doi.org/10.1111/jocn. 13455

10. Ünver S, Topçu SY, Fındık ÜY. Surgical smoke, me and my circle. Int J Car Sci [Internet]. 2016 [acessado em 10 jun. 2019];9(2):697-703. Disponível em: http://www.internationaljournalofcaringsciences. org/docs/37_Unver_original_9_2.pdf

11. Usta E, Aygin D, Bozdemir H, Uçar N. The effects of surgical smoke in operating rooms and precautions for protection. Health Sci Res. 2019;6(1):17-24. https://doi.org/10.17681/hsp.403579

12. Okgün Alcan A, Yavuz van Giersbergen M, Tanil V, Dinçarslan G, Hepçivici Z, Kurcan Ç, et al. Bir üniversite hastanesinde cerrahi duman riskleri ve koruyucu önlemlerin incelenmesi. EÜ Hemşirelik Fakültesi Dergisi [Internet]. 2017 [acessado em 10 jun. 2019];33(2):2735. Disponível em: https://dergipark.org.tr/tr/pub/egehemsire/ issue/32885/327169

13. Alp E, Bijl D, Bleichrodt RP, Hansson B, Voss A. Surgical smoke and infection control. J Hosp Infect. 2006;62(1):1-5. https://doi. org/10.1016/j.jhin.2005.01.014

14. Ball K. Surgical smoke evacuation guidelines: compliance among perioperative nurses. AORN J. 2010;92(2):e1-23. https://doi. org/10.1016/j.aorn.2009.10.026

15. Shah N.R. Commentary On: Surgical smoke - a health hazard in the operating theatre: a study to quantify exposure and a survey of the use of smoke extractor systems in UK plastic surgery units. Ann Med Surg. 2012;1:23-4. https://doi.org/10.1016/ S2049-0801(12)70008-0

16. Carvalho CRR, Toufen Junior C, Franca AS. Ventilação mecânica: princípios: análise gráfica e práticas ventilatórias. J Bras Pneumol. 2007;33(Supl. 2):54-70. http://dx.doi.org/10.1590/ S1806-37132007000800002

17. Gomes MJM. Ambiente e pulmão. J Bras Pneumol. 2002;28(5):2619. https://doi.org/10.1590/S0102-35862002000500004 
18. Mowbray N, Ansell J, Warren N, Wall P, Torkington J. Is surgical smoke harmful to theater staff? a systematic review. Surg Endosc. 2013;27(9):3100-7. https://doi.org/10.1007/s00464-013-2940-5

19. Oberdörster G, Oberdörster E, Oberdörster J. Nanotoxicology: an emerging discipline evolving from studies of ultrafine particles. Environ Health Perspect. 2005;113(7):823-39. https://doi.org/10.1289/ ehp.7339

20. Shi B. Removal of ultrafine particles by intermediate air filters in ventilations systems, evaluation of performance and analysis of applications [online PhD dissertation]. Gothenburg: Chalmers University of Technology; 2012 [acessado em 10 jun. 2019]. Disponível em: http://publications.lib.chalmers.se/records/ fulltext/165538/165538.pdf

21. Barrett WL, Garber SM. Surgical smoke: a review of the literature. Is this just a lot of hot air? Surg Endosc [Internet]. 2003 [acessado em 10 jun. 2019];17(6):979-87. Disponível em: https://doi.org/10.1007/ s00464-002-8584-5

22. Al Sahaf OS, Vega-Carrascal I, Cunningham FO, McGrath JP, Bloomfield FJ. Chemical composition of smoke produced by high-frequency electrosurgery. Ir J Med Sci [Internet]. 2007 [acessado em 10 jun. 2019];176(3):229-32. Disponível em: https://doi.org/10.1007/ s11845-007-0068-0

23. Hallmo P, Naess 0 . Laryngeal papillomatosis with papillomavirus DNA contracted by a laser surgeon. Eur Arch Otorhinolaryngol [Internet]. 1991 [acessado em 10 jun. 2019];248(7):425-7. Disponível em: https://doi.org/10.1007/bf01463570

24. Brasil. Ministério da Saúde. Secretaria de Vigilância em Saúde. Departamento de Vigilância das Doenças Transmissíveis. Guia prático sobre o HPV: perguntas e respostas. Brasília: Ministério da Saúde; 2014.
25. Fencl JL. Guideline implementation: surgical smoke safety. AORN J [Internet]. 2017 [acessado em 10 jun. 2019];105(5):488-97. Disponivel em: https://doi.org/10.1016/j.aorn.2017.03.006

26. Choi SH, Kwon TG, Chung SK, Kim TH. Surgical smoke may be a biohazard to surgeons performing laparoscopic surgery. Surg Endosc [Internet]. 2014 [acessado em 10 jun. 2019];28(8):2374-80. Disponível em: https://doi.org/10.1007/s00464-014-3472-3

27. Ulmer BC. The hazards of surgical smoke. AORN J. 2008;87(4):72134. https://doi.org/10.1016/j.aorn.2007.10.012

28. Occupational Safety and Health Administration (OSHA). Safety and Health Topics. Laser/Electrosurgery Plume [Internet]. Washington, D.C.: Occupational Safety \& Health Administration; 2015 [acessado em 18 set. 2018]. Disponível em: https://www.osha.gov/SLTC/ laserelectrosurgeryplume/

29. US Department of Labor, Occupational Safety and Health Administration, Department of Health and Human Services, Centers for Disease Control and Prevention, National Institute of Occupational Safety and Health. Hospital Respiratory Protection Program Toolkit: Resources for Respirator Program Administrators [Internet]. Estados Unidos: US Department of Labor, Occupational Safety and Health Administration; 2015 [acessado em 27 jul. 2021]. Disponível em: https://www.osha. gov/Publications/OSHA3767.pdf

30. Fencl JL. Guideline implementation: surgical smoke safety. AORN J. 2017;105(5):488-97. https://doi.org/10.1016/j.aorn.2017.03.006

31. Romano F, Gustén J, De Antonellis S, Joppolo CM. Electrosurgical smoke: ultrafine particle measurements and work environment quality in different operating theatres. Int J Environ Res Public Health Internet]. 2017 [acessado em 10 jun. 2019];14(2):137. Disponível em: https://pubmed.ncbi.nlm.nih.gov/28146089/ https://doi.org/10.3390/ijerph14020137 